\title{
PARK2 Patient Presenting with Dopa-Responsive Dystonia
}

\author{
Fumihito Yoshii $^{\text {a }}$ Koji Aono ${ }^{b}$ Ryuya Kumazawa ${ }^{c}$ Wakoh Takahashid \\ aDepartment of Neurology, Saiseikai Shonan Hiratsuka Hospital, Hiratsuka, Japan; \\ bepartment of Rehabilitation, Isehara Kyodo Hospital, Isehara, Japan; 'Department of \\ Neurology, Isehara Kyodo Hospital, Isehara, Japan; dDepartment of Neurology, Tokai \\ University Oiso Hospital, Oiso, Japan
}

Keywords

PARK2 · Dopa-responsive dystonia $\cdot{ }^{123} \mid$ FP-CIT SPECT

\begin{abstract}
We report a 34-year-old female PARK2 patient presenting with dopa-responsive dystonia (DRD). She noticed difficulty in raising her foot while walking at the age of 24 . Her lower limb symptoms were identified as dystonia later, and she was started on Menesit, which resulted in improvement of her symptoms. She was diagnosed as DRD and has been on continuous treatment since then. The specific binding ratio (SBR) of ${ }^{123}$ I FP-CIT SPECT was significantly lower than those of controls of the same age, but ${ }^{123}$ I-meta-iodobenzylguanidine myocardial scintigraphy showed a normal heart to mediastinum ratio. The Montreal Cognitive Assessment, Japanese version, was normal for her age. DRD is an inherited dystonia that typically begins during childhood and may be caused by mutations of the GCH1 (GTP cyclohydrolase), SPR (sepiapterin reductase), or TH (tyrosine hydroxylase) genes. Our patient was diagnosed as PARK2, known as autosomal-recessive juvenile Parkinson's disease, based on genetic analysis. Although there was no family history of the disease, the decrease in SBR of ${ }^{123}$ | FP-CIT SPECT enabled us to diagnose PARK2 and to differentiate this from DRD due to other genetic disorders.
\end{abstract}

\section{Karger"}




\section{Introduction}

Dopa-responsive dystonia (DRD) is an inherited dystonia that typically begins during childhood or adolescence. In most cases, dystonia appears in the lower limbs and spreads to the upper limbs over time. Symptoms may include a lack of coordination when walking or running, and patients also often develop parkinsonism. Although movement difficulties usually worsen with age, they often stabilize at around age 30 . The most common cause of DRD is mutation in the GCH1 (GTP cyclohydrolase) gene [1], or less often, mutation in the SPR (sepiapterin reductase) or TH (tyrosine hydroxylase) gene, though in some cases the cause is unknown. Depending on the genetic cause, DRD may be inherited in an autosomal-dominant or autosomal-recessive manner. This form of dystonia typically responds to treatment with low doses of levodopa and carbidopa.

PARK2 causes a form of autosomal-recessive juvenile Parkinson disease due to a mutation in the parkin protein [2]. This genetic mutation is one of the most common causes of familial PD. The most common presenting symptoms are lower-extremity dystonia and hyperreflexia, leading to gait disorders. Some patients note that their symptoms improve after sleep, although diurnal fluctuations seem to level out as the disease progresses.

We recently experienced a patient with apparent symptoms of DRD who was diagnosed as PARK2 based on genetic testing. PARK2 is often confused with DRD because of the similarity of symptoms and the effectiveness of levodopa, so here we present a case together with the findings of ${ }^{123}$ I FP-CIT SPECT (DAT scan: dopamine transporter scan) that provided the differential diagnosis.

\section{Case Presentation}

The patient is currently a 34-year-old woman. The onset was in 2011 at the age of 24, when she became aware of difficulty in raising her right foot while walking, and she visited the rehabilitation department of another hospital for the first time. After that, her right foot symptoms gradually worsened. She consulted orthopedic specialists, but no orthopedic abnormality was found. Later, she visited our neurology department, and we identified dystonia in her lower limbs. She was started on Menesit (levodopa + carbidopa) for diagnostic and therapeutic purposes and showed improvement. She was diagnosed with DRD and has been on continuous treatment since then. About 2 years later, she became pregnant, and Menesit was discontinued. After delivery, she again visited our department (September 2019). On neurological examination, she showed right lower limb dystonia, but could walk well, and retropulsion was not present. Her limb muscle tones were almost normal, and tendon reflex was reduced in both limbs. There was no tremor in her fingers, and grip power was $20 \mathrm{~kg}$ on both sides.

At that time, no abnormalities were found in blood or urine tests. Head MRI was unremarkable. ${ }^{123}$ I FP-CIT SPECT (DAT scan) gave specific binding ratio (SBR) Rt $=2.08, \mathrm{Lt}=2.26$, and these values are significantly lower than those of controls of the same age (Fig. 1) [3]. ${ }^{123}$ I-meta-iodobenzylguanidine $\left({ }^{123}\right.$ I-MIBG) myocardial scintigraphy uptake showed a normal heart to mediastinum $(\mathrm{H} / \mathrm{M})$ ratio (early $=2.79$, delayed $=3.12$, wash out ratio $=5.0 \%$ ). Cognitive assessment with Montreal Cognitive Assessment (MoCA-J), Japanese version, was almost normal (26/30) for her age.

After breastfeeding, she resumed Menesit, which improved her difficulty in moving her right leg. However, her symptoms showed diurnal fluctuations, with improvement after waking up and in the morning, but worsening in the evening. Currently, she is still receiving Menesit $200 \mathrm{mg} /$ day, which appears to be effective. Genetic screening [1] was negative for

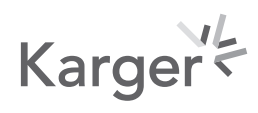




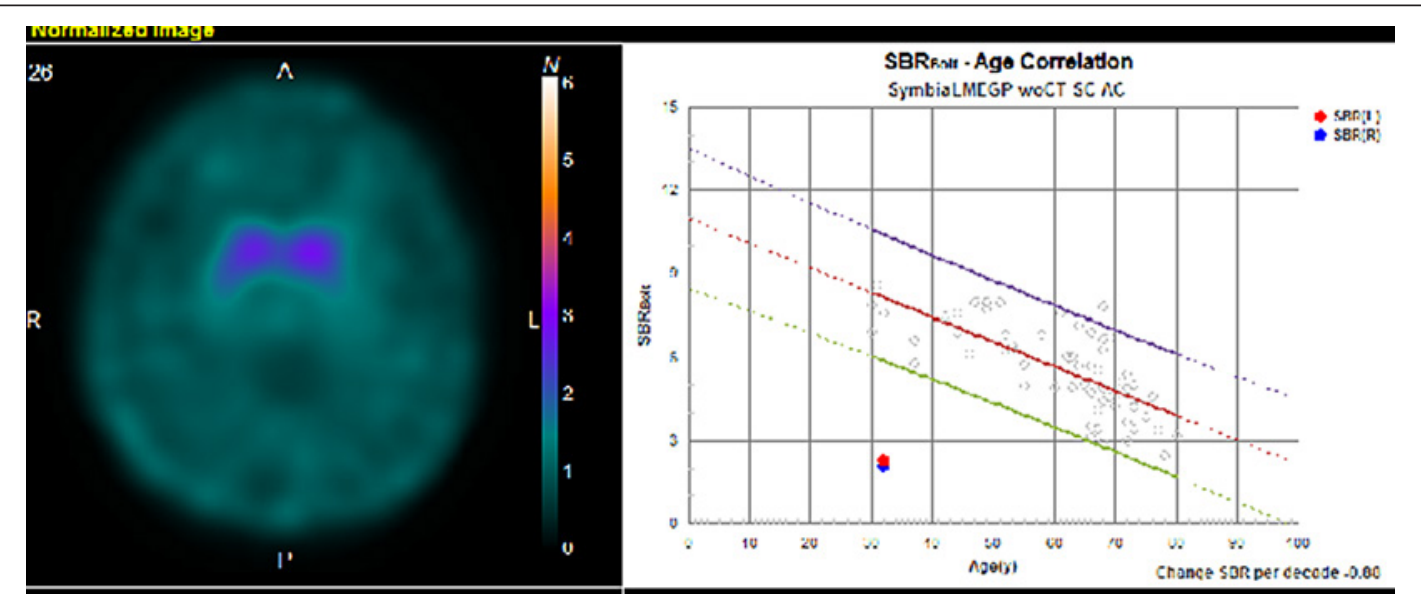

Fig. 1. ${ }^{123}$ I FP-CIT SPECT imaging of the patient (left) and comparison of her SBR values with those of normal controls by age (right).

GCH1, but PARK2 was positive (PRKN exon 3-4 heterozygous deletion, c.535-3A>G), although complete genetic screening (sequencing and quantitative analysis) was not performed. There is no family history of this disease. Therefore, family members were not genetically tested.

\section{Discussion}

Patients with DRD have selective striatonigral dopamine deficiency without neuronal cell loss in the substantia nigra, caused by genetic defects in dopamine synthesis. Dopamine is produced from tyrosine by tyrosine hydroxylase $(\mathrm{TH})$, which uses tetrahydrobiopterin (BH4) as a cofactor. A point mutation in the gene for TH has been shown to result in autosomalrecessive DRD. With regard to $\mathrm{BH} 4$ deficiencies, $>190$ different mutant alleles or molecular lesions have been identified [4], including the genes for GCH1 or SPR. However, some people with DRD do not have an identified mutation in the GCH1, SPR, or TH genes.

DRD encompasses a group of clinically and genetically heterogeneous disorders. The absence of DRD gene mutation is considered to distinguish it from young-onset PD (YOPD). Tassin et al. [5] studied 22 families with a phenotype of DRD by sequencing the GCH1 gene. Eleven heterozygous GCH1 gene mutations were identified in 12 families that included 27 patients and 13 asymptomatic carriers. Three of the remaining 10 families had deletions in the parkin gene, and no mutations were identified in 7 families. They concluded that it was difficult to distinguish, in some cases, between DRD and parkin mutations based only on the clinical spectrum. Wu et al. [6] screened 10 DRD families including 14 patients and 28 clinically unaffected relatives for GCH1, TH, and parkin genes and identified 6 novel mutations in all those genes. Their data confirmed that it is difficult to establish a clear genotype-phenotype correlation for DRD. Potsulska-Chromik et al. [7] studied 4 families clinically diagnosed with DRD. Molecular analysis revealed that the DRD phenotype was caused by a mutation in the GCH1 gene in 3 families and in the PARK2 gene in 1 family. The authors concluded that the DRD phenotype may have a heterogeneous genetic background and may be caused by point mutations or rearrangements in the GCH1 gene as well as in the PARK2 gene.

Our patient carried a heterozygous mutation in the PARK2 gene, which is considered to be a causative genetic factor for YOPD [8]. However, over the course of about 10 years, she had not shown any symptoms of parkinsonism other than dystonia in her lower limbs, and 
had no family history of autosomal-recessive inheritance of parkinsonism. In this case, the diagnosis of PARK2 rather than GCH1, TH, or SPR gene mutations might be supported by the abnormal findings of ${ }^{123}$ I FP-CIT with SPECT, which is a sensitive neuroimaging method for the assessment of nigrostriatal dopaminergic system integrity and denervation.

Naumann et al. [9] reported that ${ }^{123}$ I beta-CIT SPECT showed a striatal radiotracer uptake in the upper range of normal in a patient with DRD. This differentiates DRD from clinically similar cases of YOPD with dystonia. Jeon et al. [10] studied 5 females diagnosed as DRD based on early-onset foot dystonia and progressive parkinsonism beginning at ages 7-12. ${ }^{123}$ I beta-CIT SPECT was normal, and gene analysis showed a novel nonsense mutation in the GCHI gene in 1 family. Brajkovic et al. [11] also performed brain SPECT with ${ }^{123}$ I FP-CIT in 13 patients ( 7 males and 6 females), age 20-58 years, with a mean age of onset of 29 years, 11 patients with early-onset parkinsonian symptoms, and 2 with genetically proved DRD. Ten out of 11 patients with YOPD had decreased accumulation of ${ }^{123}$ I FP-CIT SPECT in the striatum, especially in the putamen. Two patients with initial dystonic symptoms and genetically proved DRD had normal ${ }^{123}$ I FP-CIT SPECT. These studies indicate that ${ }^{123}$ I FP-CIT SPECT is an objective neuroimaging method that is able to distinguish the neurodegenerative disease YOPD, including PARK2, from DRD not caused by a genetic abnormality of PD.

In ${ }^{123}$ I-MIBG myocardial scintigraphy, our patient showed normal early and delayed $\mathrm{H} / \mathrm{M}$ ratios despite the reduced ${ }^{123}$ I FP-CIT SPECT uptake, which is in contrast to the fact that idiopathic PD often shows decreases in both of these tests [12]. However, preserved MIBG myocardial uptake has been reported in most cases of PARK2. In postmortem examination, Orimo et al. [13] found that TH-immunoreactive nerve fibers in the epicardium were well preserved in PARK2, which would account for the normal cardiac uptake of MIBG. On the other hand, Yoshino et al. [1] reported that 7 of 7 DRD patients, harboring mutations in the GCH1 gene, presented normal H/M ratios on MIBG myocardial scintigraphy, while Yoritaka et al. [14] reported that 4 of 12 patients with PARK2 mutations exhibited decreased MIBG uptake. Therefore, the MIBG results are not always helpful in diagnosing PARK2.

Our patient showed normal cognition assessed with MoCA-J. Cognitive decline is uncommon in DRD with GCH1 mutations [1], and the cognitive impairment in carriers of parkin mutations was not different from that of other patients with early-onset PD [15]. Therefore, it is difficult to support a diagnosis of PARK2 only from the results of cognitive function tests.

\section{Conclusion}

We report a DRD patient who was diagnosed as PARK2 based on genetic analysis. Because the patient had no family history of this disease, it would be difficult to differentiate PARK2 from DRD with mutation of other genes such as TH, GCH1, or SPR based only on the clinical symptoms. ${ }^{123}$ I FP-CIT SPECT abnormalities made it possible to distinguish between these diseases.

\section{Acknowledgments}

The authors thank the patient for consenting to presentation of her data. We also thank the doctors at the Tokushima and Juntendo Universities for the genetic analysis, and Mrs. Isa Yoshinari at the Department of Neurology, Saiseikai Shonan Hiratsuka Hospital for her technical assistance.

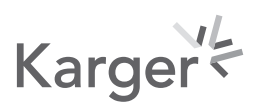




\section{Statement of Ethics}

The authors hereby declare that all work was conducted in accordance with the Declaration of Helsinki (2013), and the patient was informed of the purpose of the case presentation and gave written informed consent for publication (including publication of gene analysis results and images). Since this is a case study involving a single patient under our care, we are exempted from institutional review board approval according to our institutional review board's regulations.

\section{Conflict of Interest Statement}

The authors declare that there are no conflicts of interest regarding the publication of this article.

\section{Funding Sources}

This case report was not supported by any specific grant from funding agencies in the public, commercial, or nonprofit sectors.

\section{Author Contributions}

Fumihito Yoshii, MD, (the principal researcher) examined the patient, contributed to data collection and interpretation, and wrote the manuscript. Koji Aono, MD, and Ryuya Kumazawa, MD, examined the patient together with Dr. Yoshii and reviewed the manuscript. Wakoh Takahashi, MD, reviewed the manuscript.

\section{Data Availability Statement}

All data generated or analyzed during this study are included in this article. Further enquiries can be directed to the corresponding author.

\section{References}

1 Yoshino H, Nishioka K, Li Y, Oji Y, Oyama G, Hatano T, et al. GCH1 mutations in dopa-responsive dystonia and Parkinson's disease. J Neurol. 2018;265(8):1860-70.

2 von Coelln R, Dawson VL, Dawson TM. Parkin-associated Parkinson's disease. Cell Tissue Res. 2004;318(1): 175-84.

3 Matsuda H, Murata M, Mukai Y, Sako K, Ono H, Toyama H, et al. Japanese multicenter database of healthy controls for [123I]FP-CIT SPECT. Eur J Nucl Med Mol Imaging. 2018;45(8):1405-16.

4 Thöny B, Blau N. Mutations in the BH4-metabolizing genes GTP cyclohydrolase I, 6-pyruvoyl-tetrahydropterin synthase, sepiapterin reductase, carbinolamine-4a-dehydratase, and dihydropteridine reductase. Hum Mutat. 2006;27(9):870-8.

5 Tassin J, Dürr A, Bonnet AM, Gil R, Vidailhet M, Lücking CB, et al. Levodopa-responsive dystonia. GTP cyclohydrolase I or parkin mutations? Brain. 2000;123 (Pt 6):1112-21.

6 Wu ZY, Lin Y, Chen WJ, Zhao GX, Xie H, Murong SX, et al. Molecular analyses of GCH-1, TH and parkin genes in Chinese dopa-responsive dystonia families. Clin Genet. 2008;74(6):513-21.

7 Potulska-Chromik A, Hoffman-Zacharska D, Łukawska M, Kostera-Pruszczyk A. Dopa-responsive dystonia or early-onset Parkinson disease - genotype-phenotype correlation. Neurol Neurochir Pol. 2017;51(1): $1-6$.

\section{Karger'}


8 Li T, Kou D, Cui Y, Le W. Whole exome sequencing identified a new compound heterozygous PRKN mutation in a Chinese family with early-onset Parkinson's disease. Biosci Rep. 2020;40(5):BSR20200534.

9 Naumann M, Pirker W, Reiners K, Lange K, Becker G, Brücke T. [123I]beta-CIT single-photon emission tomography in DOPA-responsive dystonia. Mov Disord. 1997;12(3):448-51.

10 Jeon BS, Jeong JM, Park SS, Kim JM, Chang YS, Song HC, et al. Dopamine transporter density measured by [123I] beta-CIT single-photon emission computed tomography is normal in dopa-responsive dystonia. Ann Neurol. 1998;43(6):792-800.

11 Brajkovic LD, Svetel MV, Kostic VS, Sobic-Saranovic DP, Pavlovic SV, Artiko VM, et al. Dopamine transporter imaging (123)I-FP-CIT (DaTSCAN) SPET in differential diagnosis of dopa-responsive dystonia and youngonset Parkinson's disease. Hell J Nucl Med. 2012;15(2):134-8.

12 Yoshii F, Ryo M, Baba Y, Koide T, Hashimoto J. Combined use of dopamine transporter imaging (DAT-SPECT) and 123 I-metaiodobenzylguanidine (MIBG) myocardial scintigraphy for diagnosing Parkinson's disease. J Neurol Sci. 2017;375:80-5.

13 Orimo S, Amino T, Yokochi M, Kojo T, Uchihara T, Takahashi A, et al. Preserved cardiac sympathetic nerve accounts for normal cardiac uptake of MIBG in PARK2. Mov Disord. 2005;20(10):1350-3.

14 Yoritaka A, Shimo Y, Shimo Y, Inoue Y, Yoshino H, Hattori N. Nonmotor symptoms in patients with PARK2 mutations. Parkinsons Dis. 2011;2011:473640.

15 Lohmann E, Thobois S, Lesage S, Broussolle E, Tezenas du Montcel S, Ribeiro M-J, et al., French Parkinson's Disease Genetics Study Group. A multidisciplinary study of patients with early-onset PD with and without parkin mutations. Neurology. 2009;72(2):110-6. 